\title{
Designing an eHealth Coaching Solution to Improve Transitional Care of Seniors' with Heart Failure: End-User Needs
}

\author{
Jessica ROCHAT ${ }^{\mathrm{a}, \mathrm{b}, 1}$, Alexandra VILLAVERDE ${ }^{\mathrm{b}}$, Helge KLITZING $^{\mathrm{c}}$, Tore \\ LANGEMYR LARSEN ${ }^{c}$, Martin VOGEL ${ }^{\mathrm{b}}$, Jacques RIME ${ }^{\mathrm{b}}$, Ionut ANGHEL ${ }^{\mathrm{d}}$, Tudor \\ CIOARA $^{\mathrm{d}}$ and Christian LOVIS ${ }^{\mathrm{a}, \mathrm{b}}$ \\ ${ }^{\text {a }}$ Faculty of Medicine, University of Geneva, Switzerland \\ ${ }^{\mathrm{b}}$ Division of Medical Information Sciences, University Hospitals of Geneva, \\ Switzerland \\ c Seniornett Norge, Norway \\ ${ }^{d}$ Computer Science Department, Technical University of Cluj-Napoca, Romania
}

\begin{abstract}
Based on scientific studies, heart failure is the principal cause of hospitalization among seniors. More than $50 \%$ of elderly with heart failure are readmitted to hospital within six months. Readmission is linked with poor compliance with medical treatment and recommendations, emphasizing the need for a tool to help seniors better comply with post-discharge measures. The goal of this study was to identify end-user needs for the development of a coaching solution aiming to support elderly patients but also formal and informal caregivers. End-user needs were identified through interviews with the three end-user profiles: seniors with heart failure and formal and informal caregivers. The results present six categories of needs: daily treatment follow-up; healthcare network communication; transfer of information; synchronization with current digital tools; information access; and psychosocial support. The identified needs will help to develop an eHealth solution to improve care management and coaching after discharge.
\end{abstract}

Keywords. Elderly, heart failure, user-centered design, needs assessment, robotics, user-computer interface, patient discharge, patient readmission, home care services, treatment adherence and compliance, patient care, transitional care

\section{Introduction}

Although the incidence of heart failure (HF) is around 1-2\% in the common population, it reaches $10-20 \%$ among seniors [1]. Regarding these seniors patients ( $>65$ years old), $\mathrm{HF}$ is one of the principal cause of mortality and hospitalization [2,3]. More than $50 \%$ of seniors with heart failure (SHF) require readmission within 6 months after discharge [4]. Studies demonstrated that readmission is linked to a poor compliance with the treatment and lifestyle changes recommendations [4,5].

Moreover, uncoordinated episodic healthcare services that fail to patient recovery generates high rates of preventable hospitalizations [6]. Often, transitional care (TC)

\footnotetext{
${ }^{1}$ Corresponding author, Jessica Rochat, Rue Gabrielle-Perret-Gentil 4, 1205 Geneva, Switzerland; Email: jessica.rochat@hcuge.ch
} 
does not consider many of the patients' needs and personal goals during their convalescence, leading to physical and emotional stress for patients and caregivers. It is estimated that between $13 \%$ and $20 \%$ of readmissions of chronically ill older adults can be avoided by employing TC models to address patients' physical and social needs [7]. Several issues lead to difficulties when discharging patients [8-10]: not having a proper care network to support patients during convalescence; lack of knowledge of patients and informal caregivers to optimize self-management of recovery; poor adherence to post-discharge instructions; time-consuming process for healthcare professionals (HCP).

H2HCare is a European project aiming to support SHF in their transition from hospital to home by providing an eHealth robot-based coaching solution to support the post discharge treatment plan and lifestyle changes [11]. The solution intends to assist SHF but also formal (FC) and informal caregivers (IC), providing them with efficient monitoring and information transfer. The development of the H2HCare solution is following a user-centered approach [12] by involving end-users at each stage of the project. In this study, our goal is to present the user needs analysis of the three end-user profiles (SHF, FC, IC) to consider for the development of such coaching solution.

\section{Methods}

The user-centered approach allows to involve end-users during the design and development of a product to ensure that it meets end-users needs and is easy to use [12]. To identify end-users needs, semi-directive interviews were performed [13].

\subsection{Interviews}

Semi-directive interviews were conducted in two countries with three end-user profiles: 1) SHF ( $>65$ years old); 2) FCs involved in pre-discharge and/or post-discharge caring for SHF, and 3) ICs caring for a relative SHF. The interviews aimed to collect data on the activities, challenges and needs of each user profiles to understand how a robot-based solution could improve the discharge process. The interview grid contained open-ended questions to allow spontaneous answers [14] and was divided into three sections:

- General questions about the discharge process: asked to all end-users to understand each process steps and the implication of each end-user's profile.

- End-users focused questions: tailor-made questions for each end-users' groups to identify their specific needs, wishes, and priorities

- Concept-related questions: tailor-made questions for specific end-users' groups to specify predefined ideas of functionalities.

\subsection{Results analysis}

The data collected during the interviews were categorized by a thematic analysis, which consists of identifying the main units of meaning in the discourse by their presence and frequency. This classification allows to identify several categories of themes [15]. 


\section{Results}

The interviews took place from June to August 2020 with 17 participants involved in Switzerland (CH) and Norway (NO). In total, 8 SHF were involved (6 in NO, 2 in $\mathrm{CH}$ ) with an average age of 74 years, 4 FC (only in $\mathrm{CH}$ ) and 5 IC (only in NO).

The resulting end-user needs could be divided into six categories described below:

\section{Daily treatment follow-up}

For SHFs, the solution should assist with medication intake, serve as a calendar with reminders and provide motivational feedback as well as physical exercises incentives.

FCs expressed the need for functionalities such as reminders to achieve their daily tasks, centralization of all necessary information about the SHF's care, and finally emphasized that the tool should also offer an alarm system, with SHF's vital signstargeted alerts and the possibility to reach an emergency call center.

For ICs, the solution should provide a facilitated access to the current medical state and offer them the possibility to be more involved in the discharge process by accessing to the relevant medical information provided previously by HCPs.

\section{Healthcare network communication}

For SHFs and ICs, the system should provide the referring HCP's contact information, together with a mean of contact through the tool in case of a need for advice regarding follow-up recommendations or emergencies. Automatic emergency message could be sent to relevant HCPs based on monitored data.

For FCs, the tool should offer the possibility to contact the different HCPs involved in the SHF's treatment in case of problems or questions (nurses, cardiologist, attending physician, coordinators of the medico-social centre).

\section{Coordination and transfer of information}

FCs highlighted the need to have a direct transmission of the information between HCPs working in different locations. They also need more SHF's medical information (medical background, treatment history, prescriptions and physiological data). The tool could promote more transversal efforts in terms of the coordination of information. Having rapid access to such information, as well as being able to follow the different HCPs interventions (hospitalizations, medical visits, physiotherapy and home nurse visits) would allow them to be more efficient if adaptations of treatment or care are necessary.

\section{Synchronization with current digital healthcare tools}

FCs stressed that it would be efficient to have a synchronization with the various digital patient files in place (home care institution, hospital, national electronic patient record) to centralize all the information. Funnelling information sources would increase efficiency and accuracy. Currently, information present in digital systems is often incomplete which forces FCs to concatenate information from various sources within the healthcare network.

\section{Management and provision of information}

SHFs wish the system to offer them therapeutic educative information on HF such as information on disease, treatments, medication and recommendations.

SHFs and ICs ask for the possibility to have access to meaningful information about the discharge process (organization, what to expect, advices, and other resources such as events organized by the health system or associations for patients with heart failure). 
For FCs, it is important that the tool provides clear and accessible information regarding treatments, planning of care, contacts information but also administrative information linked to newfound needs (e.g. administrative procedures for the reimbursement of services such as household help). Information should be available for HCPs, but also SHF and ICs and all profiles should be able to add information.

\section{Psychosocial support}

SHF, ICs and FCs agreed that the tool should offer social and psychological support to the patient. The robot could offer a presence, act as a coach by monitoring their mood and mental health, by proposing social activities to the patients, serious games and offer the possibility to contact the relatives by video call. The tool could also provide information about external sources of support such as HF related associations.

\section{Discussion}

Compliance with treatment plan and lifestyle changes recommendations can diminish readmission of SHF $[4,5]$. We aimed to identify user needs for the development of a coaching solution to support post-discharge care management. Interviews with end-users allowed us to identify six categories of needs to meet when developing a coaching solution for TC of HF. The expressed needs required to help with the treatment followup; to simplify the communication between the SHF, the IC and the healthcare network; to improve the transmission of information among the different HCPs involved; to synchronise the new solution with current tools; to provide information about administrative procedures, the discharge process, the treatment plan and therapeutic education; and finally to offer a psychosocial support.

The main gaps of the TC process are mostly related to the poor monitoring of patients [16]. The implementation of smart environments and adoption of social robots may change this situation [17], as they enable daily remote monitoring and efficient follow-up, simplifying the communication and intervention coordination. This can improve communication among HCPs, but also set the base for implementing personalized interventions to improve patient engagement and adherence to the postdischarge therapy [18]. Efforts to avoid readmission usually start in the emergency department prior to patient discharge and continue in ambulatory departments [19]. Most of pre-discharge assessment models don't take a longitudinal view of patients [20], and don't use this information to provide a treatment plan and therapeutic education.

In our view this can be improved by providing the necessary support to conduct whole person assessment before discharge to identify readmission risks and use this information to configure and personalize the TC using social robots. The resulting needs were used to define functionalities and design first mock-ups of the solution for digitizing the TC process to proactively detect problems leading to re-hospitalization and deliver coordinated and personalized interventions. Although sample size in qualitative studies is continually discussed [21], the recommended number of participants for phenomenological studies ranges around 6-10 per studied group [22]. Our sample size may not have been sufficient to reach saturation, emphasizing the importance of iterative involvement of end-users in the design and evaluation processes [23]. 


\section{Funding}

This research was funded by InnoSuisse, the Research Council of Norway and the Romanian National Authority for Scientific Research and Innovation with co-funding from the European Union's Horizon 2020 and the Active Assisted Living programs.

\section{References}

[1] Roger VL. Epidemiology of Heart Failure. Circ Res. 2013 Aug 30;113(6):646-59.

[2] Butrous H, Hummel SL. Heart Failure in Older Adults. Vol. 32, Canadian Journal of Cardiology. Pulsus Group Inc.; 2016. p. 1140-7.

[3] Braunwald E. The war against heart failure: The Lancet lecture. Vol. 385, The Lancet. Lancet Publishing Group; 2015. p. 812-24.

[4] Ogbemudia EJ, Asekhame J. Rehospitalization for heart failure in the elderly. Saudi Med J. 2016;37(10):1144-7.

[5] Van Der Wal MHL, Jaarsma T, Van Veldhuisen DJ. Non-compliance in patients with heart failure; How can we manage it? Vol. 7, European Journal of Heart Failure. John Wiley \& Sons, Ltd; 2005. p. 5-17.

[6] Oster A, Bindman AB. Emergency Department Visits for Ambulatory Care Sensitive Conditions. Med Care. 2003 Feb;41(2):198-207.

[7] Finlayson K, Chang AM, Courtney MD, Edwards HE, Parker AW, Hamilton K, et al. Transitional care interventions reduce unplanned hospital readmissions in high-risk older adults. BMC Health Serv Res. 2018 Dec $12 ; 18(1)$.

[8] Albrecht JS, Gruber-Baldini AL, Hirshon JM, Brown CH, Goldberg R, Rosenberg JH, et al. Hospital Discharge Instructions: Comprehension and Compliance Among Older Adults. J Gen Intern Med. 2014 Nov 1;29(11):1491-8.

[9] Fuji KT, Abbott AA, Norris JF. Exploring Care Transitions From Patient, Caregiver, and Health-Care Provider Perspectives. Clin Nurs Res. 2013 Aug 30;22(3):258-74.

[10] Plank A, Mazzoni V, Cavada L. Becoming a caregiver: new family carers' experience during the transition from hospital to home. J Clin Nurs. 2012 Jul 1;21(13-14):2072-82.

[11] H2HCare AAL - Social robot-based solution for elders' care management and coaching after discharge from hospital to home. Available from: https://h2hcare-aal.eu/

[12] Dabbs ADV, Myers BA, Mc Curry KR, Dunbar-Jacob J, Hawkins RP, Begey A, et al. User-centered design and interactive health technologies for patientsi. CIN - Comput Informatics Nurs. 2009 May;27(3):175-83.

[13] Lallemand C, Gronier G. Méthodes de Design UX. 30 méthodes fondamentales pour concevoir et évaluer les systèmes interactifs. Eyrolles.; 2016.

[14] Züll C. Open-Ended Questions. GESIS Surv Guidel. 2016;3.

[15] Fallery B, Rodhain F. Quatre approches pour l'analyse de données textuelles: lexicale, linguistique, cognitive, thématique. AIMS; 2013.

[16] Pollack AH, Backonja U, Miller AD, Mishra SR, Khelifi M, Kendall L, et al. Closing the gap: Supporting patients' transition to self-management after hospitalization. In: Conference on Human Factors in Computing Systems - Proceedings. Association for Computing Machinery; 2016. p. 5324-36.

[17] Anghel I, Cioara T, Moldovan D, Antal M, Pop CD, Salomie I, et al. Smart environments and social robots for age-friendly integrated care services. Int J Environ Res Public Health. 2020 Jun 1;17(11).

[18] Rennke S, Ranji SR. Transitional Care Strategies From Hospital to Home: A Review for the Neurohospitalist. The Neurohospitalist. 2015;5(1):35-42.

[19] Kripalani S, Theobald CN, Anctil B, Vasilevskis EE. Reducing hospital readmission rates: Current strategies and future directions. Vol. 65, Annual Review of Medicine. Annual Reviews Inc.; 2014. p. 471-85.

[20] Dykes PC, Samal L, Donahue M, Greenberg JO, Hurley AC, Hasan O, et al. A patient-centered longitudinal care plan: Vision versus reality. J Am Med Informatics Assoc. 2014;21(6):1082-90.

[21] Dworkin SL. Sample size policy for qualitative studies using in-depth interviews. Vol. 41, Archives of Sexual Behavior. Springer Science and Business Media, LLC; 2012. p. 1319-20.

[22] Marshall B, Cardon P, Poddar A, Fontenot R. Does sample size matter in qualitative research?: A review of qualitative interviews in is research. J Comput Inf Syst. 2013;54(1):11-22.

[23] Nielsen J. The Usability Engineering Life Cycle. Computer (Long Beach Calif). 1992;25(3):12-22. 